\title{
Los usuarios y las webs de los archivos históricos nacionales: el caso del Arquivo Nacional da Torre do Tombo (Portugal)*
}

\author{
Amalia Más Bleda \\ Celia Chaín Navarro **
}

Artículo recibido:

18 de diciembre de 2008.

Artículo aceptado:

26 de marzo de 2009.

\section{RESUMEN:}

El objetivo de este artículo es conocer cuál es la información o los servicios que se deberían incluir en el sitio web de un archivo, tomando como referencia la opinión de su público. Para ello analizamos las necesidades de 90 usuarios de un archivo concreto: el Arquivo Nacional da Torre do Tombo (Archivo Nacional de Portugal), obtenidas mediante la utilización de una entrevista personal. Se concluye que la mayoría de los usuarios desean encontrar en el sitio web información básica o práctica, como datos de contacto y condiciones de acceso y consulta, así como una descripción detallada de los fondos

\footnotetext{
Este trabajo es uno de los resultados del proyecto de investigación I+D+i subvencionado por la Secretaría General de Política Científica y Tecnológica del Ministerio de Ciencia e Innovación con referencia HUM-2007-61093/HIST.

** Ambas autoras pertenecen a la Universidad de Murcia, España. (Amalia: amaliamas@um.es); (Celia: chain@um.es)

INVESTIgACIÓN BIBLIOTECOLÓGICA, Vol. 23, Núm. 47, enero/abril, 2009, México, ISSN: 0187-358X. pp. 189-212
} 
que custodia el archivo, el mayor número posible de documentos digitalizados, un buen sistema de búsqueda (catálogo) y la posibilidad de hacer trámites en línea.

Palabras clave: Estudio de usuarios, necesidades de información, sitio web, sede web, Internet, Archivo Nacional, Portugal, contenidos digitales, satisfacción del usuario, servicios en línea.

\begin{abstract}
Webs and national archives users: the case of Arquivo Nacional de Torre do Tombo (Portugal)

Amalia Más Bleda and Celia Chaín Navarro

Information is given of services that should be included in the website of an archive, taking as reference the opinion of its public. Therefore the needs of 90 users of this concrete archive, the Arquivo Nacional da Torre do Tombo (National Archive of Portugal) are analyzed, which were obtained through a personal interview. Conclusions are that most users wish to find basic or practical information in the web, such as contact, access and consultation data; a detailed description of the archive's funds; the number of digitalized documents on-line; a good search system (catalog), and the possibility of doing transactions on-line.
\end{abstract}

Keywords: Users' study; Information needs, Websites; Web sites; Internet; National Archive; Portugal; Digital contents; Users' satisfaction; On-line services.

\title{
I. INTRODUCCIÓN
}

\footnotetext{
Cualquier producto o servicio debe diseñarse desde la perspectiva de los $\checkmark$ usuarios que lo utilizarán. Esto es aplicable tanto en las instituciones públicas como en las privadas, aunque parece que las instituciones privadas han tomado conciencia de ello más rápidamente, quizás porque el beneficio económico está en relación directa con su capacidad para satisfacer las necesidades de sus clientes. Por tanto, cuando se crea un producto o servicio,
} 
el primer paso debería consistir en conocer quién es el público que lo va a utilizar y cuáles son sus necesidades con respecto a él. De lo contrario, las probabilidades de éxito pueden reducirse considerablemente.

En el presente trabajo, el producto o servicio es el sitio web de un Archivo $\mathrm{Nacional}^{1}$, y el público las personas que lo van a utilizar. Pero la pregunta clave es: ¿quiénes son esas personas?, o lo que es lo mismo ¿qué tipo de usuarios pueden hacer uso del sitio web de un archivo?

Lara y Ruiz ${ }^{2}$ distinguen al público del sitio web de un archivo como: usuario interno (aquel que tradicionalmente utiliza el archivo), ${ }^{3}$ usuario potencial (aquel que por su formación podría utilizar el servicio de archivo y no accede a él, en general, por condicionamiento de tipo práctico, social, económico), pre-usuario (gran masa de ciudadanos que quedan al margen de los circuitos culturales al uso y viven en cierta manera desvinculados de las propuestas culturales de su entorno) y no-usuario (parte de la ciudadanía que carece de las bases mínimas que le posibilitan el acceso al archivo).

Entre las categorías de los que ya son usuarios podemos hacer cuatro grandes grupos: ${ }^{4}$

1. Los investigadores profesionales o científicos. Son los usuarios tradicionales y habituales del archivo, con gran formación académica.

2. Los investigadores aficionados. Frecuentemente buscan datos concretos, suelen ser usuarios ocasionales.

3. Los profesores y estudiantes de enseñanza primaria, secundaria y universitaria. Son los que entran en contacto con el archivo por diferentes motivos, casi siempre relacionados con trabajos encargados en sus respectivos

1 Consideramos que el sitio web de un archivo puede considerarse tanto producto como servicio. Producto porque siguiendo la definición de Torrés (Antoni Torrés Rosell. Márquetin y archivos. Propuestas para una aplicación del márquetin en los archivos. Gijón: Trea, 2006, p. 142), podemos tomar el sitio web del archivo como algo que se ofrece a un público con la finalidad de que sea utilizado, con el objeto de satisfacer una necesidad. Servicio, porque el sitio web es algo intangible, no se puede tocar. Según el autor, "la utilización de páginas web, siempre y cuando éstas vayan más allá de una mera difusión del archivo, también deberán considerarse como productos específicos" (p. 142).

2 Pablo Lara Navarra y Antonio Ruiz Rodríguez. "La difusión de información en los archivos: páginas web”, en Tria. Revista de la Asociación de Archiveros de Andalucía. 1999, n. 6, p. 159.

3 Dependiendo de los autores y del marco conceptual en el que se desarrolle, también se denomina usuario externo. Si incluimos a los profesionales que trabajan en el Archivo en el conjunto de usuarios de los portales, entonces los investigadores habituales también se suelen llamar "usuarios externos", mientras que los trabajadores se consideran usuarios internos. Excluyendo del concepto a los archiveros y resto de trabajadores, entonces pueden llamarse usuarios internos.

4 Sintetizado de la ponencia de: María Rosa de Muñoz. "Usuarios internos y externos en los archivos administrativos de Panamá”, en: Seminário Internacional de Arquivos de Tradição Ibérica [en línea], 3 a 7 de abril de 2000 - Río de Janeiro. [Consulta: 20-11-2008]. Disponible en <http://www.conarq. arquivonacional.gov.br/Media/publicacoes/ibericas/usuarios_ internos_y_externos_en_los_archivos_administrativos_de_panama.pdf $>$. 
centros docentes o puede tratarse de profesores que desean mejorar su docencia.

4. Archiveros y otros profesionales de la información. Son los que buscan ideas o documentos concretos.

5. Los ciudadanos en general. Los que por diferentes motivos desean consultar los fondos pero que llegan a ellos por casualidad o por curiosidad.

Todos estos usuarios son también potenciales internautas que en un momento determinado pueden consultar la web del Archivo.

Como los usuarios suelen ser distintos se debe tener en cuenta que la existencia de un sitio web común para todos los archivos, cualquiera que sea su tipología, no es posible. Puede que parte de la información proporcionada sea la misma (todos incluyen información de contacto, por ejemplo), pero en general, el sitio web de cada archivo, así como los servicios prestados, serán diferentes. Como ejemplo podemos comparar el sitio web de un archivo municipal y el de un archivo parlamentario. Es posible que en el primero encontremos información de carácter general y con una terminología sencilla, mientras que en el segundo los fondos sean de otra temática y con una terminología especializada, donde los usuarios conocen el lenguaje empleado, así como la forma de organizar la información proporcionada. La documentación de los fondos de cada uno de los archivos es diferente, así como sus usuarios y, en consecuencia, el sitio web también lo será.

Es preciso diseñar sitios web centrados en el usuario y para ello resulta necesario conocerlos. La Interacción Persona-Ordenador (IPO), conocida también como Human Computer Interaction (HCI), es una disciplina que estudia la relación entre las personas y los ordenadores. Las investigaciones realizadas en este campo de estudio pueden resultar de gran utilidad a la hora de diseñar sitios web. Una de las disciplinas que más influencia tiene en la IPO es la Psicología Cognitiva, la cual estudia la forma en que las personas procesamos la información recibida. Otras disciplinas que ayudan a comprender mejor la parte humana de la IPO son la Filosofía, Psicología, Fisiología, Medicina y Ergonomía. ${ }^{5}$

Además existen herramientas que nos permiten obtener gran cantidad de información sobre el comportamiento de las personas que visitan un sitio web. Es el caso de los ficheros log. Son "extensos ficheros de texto plano que 
genera el servidor web, y en los que se registra cada una de las peticiones de páginas realizadas por los clientes al servidor" ${ }^{6}$

Mediante el análisis de estos ficheros se pueden responder preguntas tales como: ¿Quién usa el sitio? ¿Cuándo lo usa? ¿Qué páginas suelen ser las más visitadas? ¿Desde qué páginas se llega? ¿Qué términos utiliza el usuario para interrogar al buscador interno?, etcétera. ${ }^{7}$ En el caso de los archivos nos interesa, además, conocer información relativa al número de consultas realizadas, los principales temas de esas consultas, país desde el que está conectado el usuario, etcétera. El análisis de estos datos permite conocer mejor a los usuarios y así poder satisfacer sus necesidades de información con una mayor tasa de éxito.

Los estudios de usuarios son instrumentos de enorme utilidad para obtener gran cantidad de información sobre éstos. En palabras de $\mathrm{Sanz}^{8}$ son

el conjunto de estudios que tratan de analizar cualitativa y cuantitativamente los hábitos de información de los usuarios, mediante la aplicación de distintos métodos, entre ellos los matemáticos (principalmente los estadísticos), a su consumo de información.

Conceptos relacionados son también información, conocimiento, necesidad de información, deseo de información, demanda de información, uso de información y requerimiento de información. ${ }^{\text {? }}$

Martín ${ }^{10}$ hace un breve repaso por la historia de los estudios de usuarios y describe los distintos métodos y técnicas de recogida de datos, mientras que Sanz y Martín, ${ }^{11}$ una década antes, resaltaban la importancia de la utilización de técnicas bibliométricas para la realización de este tipo de estudios. Por su parte Hernández ${ }^{12}$ destaca la necesidad de realizar estudios previos al desarrollo de un programa de formación de usuarios, mientras que Rubio ${ }^{13}$ afirma

6 Yusef Hassan Montero, Francisco Jesús Martín Fernández e Ghzala Iazza. "Diseño Web Centrado en el Usuario: Usabilidad y Arquitectura de la Información”, en Anuario Hipertext.net [en línea]. 2004, n. 2. [Consulta: 01-10-2008]. Disponible en <http://www.hipertext.net/web/ pag206.htm >.

7 Ibidem.

8 Elías Sanz Casado. Manual de estudios de usuarios. Madrid: Fundación Germán Sánchez Ruipérez, 1994, p. 31.

9 Ibidem

10 Carmen Martín Moreno. "Metodología de investigación en estudios de usuarios”, en Revista General de Información y Documentación. 2007, vol. 17, n. 2, p. 129-149.

11 Elías Sanz Casado y Carmen Martín Moreno. "Técnicas bibliométricas aplicadas a los estudios de usuarios", en Revista General de Información y Documentación. 1997, vol. 7, p. 41-68.

12 Patricia Hernández Salazar. "La relación entre los estudios y la formación de usuarios de la información”, en Revista General de Información y Documentación. 2007, vol. 17, n. 2, p. 103-121.

13 Alfonso Rubio Hernández. "Estudios de usuarios en archivos municipales: una aproximación teórico-práctica”, en Scire. Representación y Organización del Conocimiento. 2004, vol. 10, n. 1, p. 217-236; 
que los estudios de usuarios de un archivo deben cumplir con el requisito de ocuparse de todos los tipos potenciales de usuarios.

En los últimos años se ha podido apreciar una preocupación por promover la difusión de los archivos. Estamos convencidas de que el sitio web es una de las herramientas más eficaces para lograrlo. Sin embargo, no podemos considerarlo como un lugar más dónde ofrecer información del archivo, sino como una herramienta para mejorar la comunicación entre la institución y los ciudadanos. Por ello, el sitio web debe estar centrado en los usuarios y en este sentido Hassan ${ }^{14}$ identifica la satisfacción de éstos como elemento clave para el fracaso o éxito del sitio web, además indica que entre las variables que condicionan esta satisfacción destaca el diseño del sitio web, concluyendo que la usabilidad es el factor vertebral del diseño orientado a la satisfacción-no frustración de uso. El autor diferencia entre "factores higiénicos (u orientados a la no frustración) y factores motivadores (orientados a la satisfacción), destacando la usabilidad como factor motivador e higiénico simultáneamente". Entre los que él denomina factores higiénicos se encuentran la funcionalidad, accesibilidad y findability; y entre los llamados motivadores, la utilidad, estética y credibilidad.

Relacionados con los estudios de usuarios y los sitios web se encuentran los servicios de referencia virtual, puesto que éstos son uno de los servicios que pueden (y deberían, en la medida de sus posibilidades) ofrecer un archivo a través de un sitio web. Y para ello también resulta necesario conocer las necesidades de los usuarios que van a utilizar este servicio.

El servicio de referencia virtual se puede considerar como aquel que, mediante el empleo de las tecnologías de la información, permite mejorar la comunicación entre los usuarios y los profesionales de la información. Según Manso ${ }^{15}$ este proceso está integrado por cuatro elementos fundamentales: los usuarios, la interfaz de comunicación, el profesional de la información y las fuentes de información. ${ }^{16}$

La mayoría de las obras consultadas asocian los servicios de referencia con la biblioteca, y hacen referencia a ésta en sus definiciones. Sin embargo,

14 Yusef Hassan Montero. "Factores del diseño web orientado a la satisfacción y no-frustración de uso", en Revista Española de Documentación Científica. 2006, vol. 29, n. 2, p. 239-257.

15 Ramón Alberto Manso Rodríguez. "Servicio de referencia virtual: teoría y práctica en torno a las políticas para su gestión y desarrollo", en Revista Española de Documentación Cientifica. 2008, vol. 31, n. 1, p. 39-51.

16 Una obra muy interesante (y bastante más amplia) relacionada con el servicio de referencia virtual, en la que se exponen conceptos fundamentales de este servicio, herramientas tecnológicas que se pueden aplicar a él, así como criterios para su evaluación, entre otras cosas, es: Ramón Alberto Manso Rodríguez. Servicio de Referencia Virtual: concepto, organización y evaluación [en línea]. Santa Clara (Cuba): Editorial Feijoo, 2006. [Consulta: 05-11-2008]. Disponible en <http://eprints.rclis.org/archive/00015079/>. 
estos servicios también pueden aplicarse al mundo de la archivística. No es exclusivo de las bibliotecas,

es un servicio cuya presencia nos habla de instituciones (biblioteca, museos, centro de documentación, etcétera) con real vocación de servicio que intentan 'ir hacia' el usuario con lo que tienen, poco o mucho. ${ }^{17}$

En este sentido, Navarro ${ }^{18}$ afirma que

estas herramientas de información y referencia en el campo archivístico suponen un paso adelante en la utilización del entorno web, puesto que no se trata simplemente de volcar la información sobre el archivo, los horarios de apertura, las características de sus fondos o la propia información contenida en los instrumentos de descripción. El servicio de referencia archivístico busca establecer canales de comunicación en línea con sus usuarios, permitiendo unos modos de acceso a la descripción de los documentos de archivo muy útiles antes de la definitiva y necesaria consulta in situ del documento original en el propio centro.

Aunque en la creación de un sitio web influyen muchos aspectos a tener en cuenta (usabilidad, accesibilidad, arquitectura de información, navegación, etcétera), en este trabajo nos centramos únicamente en los contenidos. Es decir, intentamos indicar, analizando la opinión de los propios usuarios, cuáles son la información y los servicios que se deberían incluir en el sitio web de un archivo.

\section{2. ОвJETIVo}

El objetivo principal de este estudio consiste en identificar la información y servicios que se deben proporcionar en la sede web de un Archivo Nacional, basándonos en la opinión de los usuarios reales de un archivo concreto: el Arquivo Nacional da Torre do Tombo (Archivo Nacional de Portugal), con sede física en la ciudad de Lisboa. ${ }^{19}$

17 Fernanda Rodríguez Briz. Los servicios de referencia virtual: surgimiento, desarrollo y perspectivas a futuro. Buenos Aires: Alfagrama, 2005, p. 138.

18 Diego Navarro Bonilla. "El servicio de referencia archivístico: retos y oportunidades", en Revista Española de Documentación Cientifica. 2001, vol. 24, n. 2, p. 191.

19 Este trabajo surgió con ocasión de una estancia en dicho Archivo financiada por el Ministerio de Ciencia e Innovación español durante los meses de junio a septiembre de 2008. 


\section{Metodología}

Como ya se ha mencionado, pretendemos conocer cuáles son las necesidades informativas de los usuarios de un Archivo Nacional con respecto a su sitio web. Para ello se ha realizado una breve entrevista personal (en portugués) a los usuarios reales de un archivo concreto, el Arquivo Nacional da Torre do Tombo (en adelante ANTT). Este sitio web se encuentra en periodo de reestructuración (figura 1).

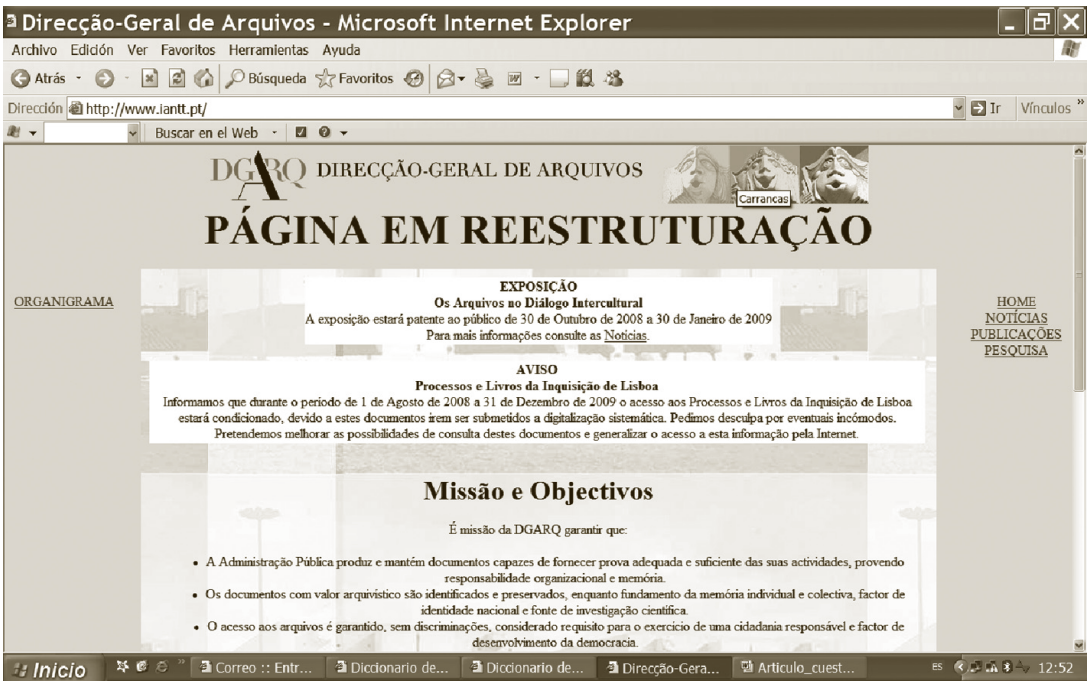

Fig. 1. Web en reestructuración del Archivo Nacional Torre do Tombo (10-12-2008)

El Archivo Nacional de la Torre do Tombo (Arquivo Nacional da Torre do Tombo), cuya historia se remonta a finales de la Edad Media, es una de las instituciones portuguesas activas más antiguas. El origen de este nombre se debe a que el archivo estuvo instalado, desde cerca de 1378 hasta 1755 en una torre en el Castillo de San Jorge (Castelo de São Jorge), en la ciudad de Lisboa, llamada Torre do Tombo. Durante la mayor parte del siglo XX, mantiene su papel de Archivo Nacional y del principal archivo del país, pero institucional y funcionalmente desligada al sistema archivístico nacional. Entre 1997 y 2006, se denomina oficialmente Instituto dos Arquivos Nacionais/Torre do Tombo (IANTT), y depende del Ministerio de Cultura. A partir del año 2006 el Archivo Nacional da Torre do Tombo se integra en la estructura de la nueva Direç̧ão-Geral de Arquivos (DGARQ). Por este motivo se está llevando a cabo la reestructuración del sitio web. ${ }^{20}$ 
Sus fondos ocupan un espacio cercano a los 70 kilómetros. El documento más antiguo, una carta de fundación de una iglesia en Lordosa, data del año 882, aunque también se encuentran otros tesoros como a Bula Manifestis Probatum (1179), que confirmó internacionalmente a Portugal como Reino independiente.

Los fondos, ${ }^{21}$ de indudable riqueza y cuyo contenido es difícil de sintetizar, recogen documentación histórica sobre la casa real portuguesa, nobleza, cuentas del reino, Inquisición, antiguas colonias, compañías de comercio, asientos, salud pública, legislación y crónicas. De las instituciones contemporáneas conservan documentos procedentes de los Ministerios de Hacienda, Finanzas, Interior y Consejo de Estado.

\subsection{Puntos de partida para la recogida de datos y la orientación del trabajo}

Estudios previos a éste nos hicieron analizar la posibilidad de preguntar a los usuarios sobre la información que les gustaría encontrar en la página web de un Archivo. Es sabido que algunos investigadores y usuarios no son demasiado partidarios del uso de las tecnologías de la información y de la comunicación (TIC), tradicionalmente el número mas elevado de éstos se encuentra entre los investigadores del área de las ciencias sociales y humanidades y entre las personas que tienen más edad. Por ello era básico averiguar las opiniones de los usuarios actuales del Archivo con respecto a varias cuestiones, previas a conocer su opinión sobre la información que debe aparecer en el sitio web. Primeramente era necesario saber si existía un acuerdo respecto a los instrumentos de búsqueda existentes y si consideraban que éstos eran adecuados, ya que la herramienta de consulta de los fondos es lo primero que habitualmente encontramos, tanto cuando se acude de forma presencial como en la página web de cualquier institución documental. En un segundo análisis era necesario conocer si manifestaban interés en consultar la información del

21 Entre los fondos y colecciones sobre el Antiguo Régimen destacan los provenientes de la Casa de la Corona y de la Casa Real, Cancillería Regia, Cuentas del Reino y del Estado de la India, Cortes, Leyes y Ordenanzas, Bulas, Tratados, Colecciones de Cartas, Crónicas, Libros de Linajes, Colegio de Nobles, etcétera. De la Administración Central sobresalen la Casa de las Súplicas, Inquisición de Lisboa, de Coimbra y de Évora, Cancillerías, Hacienda, Casa de Cuentas del Reino, Consejo de Guerra, Consejo Ultramarino, Junta de los Tres Estados, Junta da Administración del Tabaco, Real Academia de Historia, Secretaría de Estado de Negocios del Reino, Junta del Comercio, Real Fábrica de Sedas, Compañías de comercio transatlánticas, Junta de Liquidación de fondos, Intendencia General de Policía, Real Mesa de Censura, Extracción de Diamantes de las Minas de Brasil, Junta de Salud Pública, Casa de la Moneda, Proveedurías, Gobierno del Estado de la India, Casa del Infantado y Hospital de San José. Referido a Instituciones Contemporáneas, contiene documentación del Consejo de Estado, Ministerio de Hacienda y Finanzas, y Ministerio del Interior. También cartas y colecciones particulares de ciudadanos ilustres, genealogías manuscritas, una importante colección cartográfica y de Constituciones Políticas. 
Archivo fuera de la institución, y si estaban dispuestos a hacerlo a través de la página web de éste.

Para conocer datos sobre las primeras cuestiones nos remitimos a estudios previos. Así, en uno realizado en el año 2005 sobre las prioridades de mejora establecidas por los usuarios del ANTT (figura 2), de las 10 señaladas, 7 están directamente relacionadas con el acceso a la información del Archivo (las que aparecen subrayadas), lo que evidencia la importancia que tiene para cualquier usuario la facilidad y rapidez de acceso a instrumentos que faciliten la consulta sobre la información archivística de forma sencilla, precisa, pertinente, adecuada, segura y con múltiples posibilidades. Todo ello se refiere, pues, a la presentación de contenidos que perfectamente pueden derivarse hacia servicios vía Internet a través de las páginas web de los archivos.

\begin{tabular}{|l|}
\multicolumn{1}{|c|}{ Prioridad Máxima } \\
\hline - Precisión en los instrumentos de búsqueda documental \\
- Tiempo de espera para obtención de los servicios / productos \\
- Diversidad en las formas de acceso a servicios / información / productos \\
- Tiempo de espera para entrega de los documentos para consulta \\
- Capacidad auto explicativa de los instrumentos de búsqueda documental \\
- Precios razonables \\
\hline \multicolumn{2}{c|}{ Prioridad Media } \\
\hline $\begin{array}{l}\text { - Rapidez en la obtención de información a través de la consulta de los instrumentos de búsqueda documen- } \\
\text { - } \text { Fal }\end{array}$ \\
\hline - Competildad de acceso a la información sobre los servicios / productos \\
\hline Prioridad Mínima \\
\hline - Consistencia y pertinencia de la información
\end{tabular}

Fig. 2: Prioridad que establecen los usuarios del Archivo Nacional de la Torre do Tombo para la mejora de los servicios prestados.

Fuente: Arquivo Nacional da Torre do Tombo. Sondagem à Satisfação dos Clientes 2005. Relatório Final. Realizado por José Maria Furtado y Sérgio Nuno Leitão Barreiros. Lisboa: Instituto dos Arquivos Nacionais - Torre do Tombo, 2006, p. 9

Para seguir recogiendo datos del triángulo formado por los conceptos relacionados con necesidades de información, posibilidades de acceso a ella y satisfacción de usuarios, es muy conveniente conocer la opinión que éstos tienen sobre las mejores formas de acceder a las herramientas de descripción de los fondos, ya que a ese respecto el sitio web es sólo una de esas posibilidades. Tomamos los datos publicados por el ANTT en el año 2006 sobre la forma que los usuarios prefieren (figura 3). El sistema que tenía mayores partidarios era Internet (casi un 40\%) ya en 2005, año en el que se realizó la encuesta. La búsqueda manual, realizada a través de los sistemas tradicionales, es considerada como la más adecuada por algo más de la cuarta parte de los usuarios 
(28,3\%). La concienciación sobre las ventajas y posibilidades que ofrece la información en soporte electrónico (ya sea a través de la red como en bases de datos consultadas en la propia institución archivística) supera, según la tabla, el 65\%. Ello significa que la información electrónica es, para más de la mitad de los usuarios del Archivo, la forma más adecuada de realizar una búsqueda sobre los fondos de la institución.

\begin{tabular}{|l|c|}
\hline \multicolumn{1}{|c|}{ Instrumento de búsqueda más adecuado } & $\%$ \\
\hline Manual & 28,3 \\
\hline Electrónica (Bases de Dados in situ) & 26,2 \\
\hline Internet & 39,3 \\
\hline Todas & 6,2 \\
\hline Total & 100 \\
\hline
\end{tabular}

Fig. 3: Soportes más adecuados para facilitar búsqueda documental, según los usuarios del Archivo Nacional de la Torre do Tombo.

Fuente: Arquivo Nacional da Torre do Tombo. Sondagem à Satisfação dos Clientes 2005. Relatório Final. Realizado por José Maria Furtado y Sérgio Nuno Leitão Barreiros. Lisboa: Instituto dos Arquivos Nacionais - Torre do Tombo, 2006, p. 14 del Apéndice II.

Evidenciada la aceptación que por parte de los usuarios tiene la búsqueda virtual, faltaba actualizar los datos y recoger opiniones referentes a la obtención de otro tipo de información, productos o servicios a través del sitio web del Archivo, y no sólo de los instrumentos de descripción documental. Antes de ello es necesario explicar cómo funcionaba la web del ANTT y las características y servicios que tenía, con objeto de que se puedan entender mejor y situar adecuadamente las opiniones vertidas por los usuarios de esta institución.

En el sitio web del Arquivo Nacional da Torre do Tombo (actualmente en reestructuración) se ofrecían un instrumento de consulta (figura 4) en el que se recogían las descripciones relativas a los fondos y colecciones existentes en el Archivo, con indicaciones sobre su procedencia, forma de constitución, ámbito cronológico, contenido, etcétera; así como los instrumentos de descripción disponibles en la Sala de Referencia. Permitía dos formas de consulta: 1) introduciendo los términos de consulta deseados y 2) búsqueda por orden alfabético. Desde esa misma página web, se informaba al usuario que la solicitud para la consulta de documentos se podía hacer por teléfono y correo electrónico (con la condición de que el lector proporcionara su identificación completa y el número de tarjeta), así como a través de un formulario electrónico.

Además, en el sitio web también se ofrecía una guía abreviada (en los formatos de Word y en .pdf) de las Instituciones del Antiguo Régimen, Instituciones 

Singulares, Asociaciones, Empresas e Instituciones Eclesiásticas.

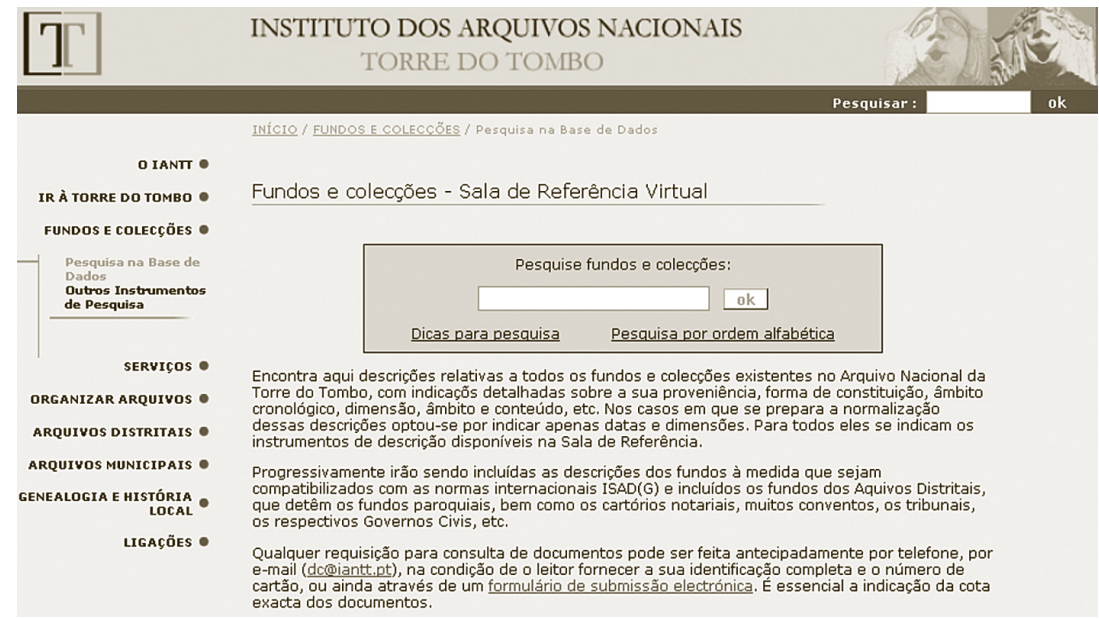

Fig. 4: Instrumento de búsqueda ofrecido en el website antiguo del Archivo Nacional de la Torre do Tombo

\subsection{La entrevista}

Para ello se hicieron un total de 90 entrevistas, entre los meses de julio y septiembre del año 2008, a los usuarios que se encontraban en la sala de consulta de dicho Archivo.

La entrevista estaba compuesta por dos partes. En la primera se le pedía al usuario que nos dijera qué información o servicios le gustaría que se incluyera en el sitio web del archivo. En la segunda se le preguntaba por su nacionalidad y profesión. Mientras el usuario expresaba sus respuestas, la entrevistadora las iba anotando. También se anotaba el género y edad del usuario. Se establecieron tres rangos: menos de 40 años, entre 40 y 60 años y más de 60 años.

El primer día se realizó la entrevista a 18 usuarios y se preguntó qué información, servicios o productos les gustaría que se incluyeran en el sitio web. Finalizada la primera sesión consideramos interesante conocer también datos de los usuarios relativos a la nacionalidad, profesión, sexo y edad, especialmente por si era posible establecer diferencias entre esos grupos. De la mayoría de los usuarios entrevistados pudimos obtener todos los datos.

La entrevista se confeccionó con preguntas de respuesta abierta, de forma que los usuarios pudieran expresar sus opiniones libremente, y en ningún caso se les dio pista alguna para no influir en sus respuestas. 


\section{Resultados}

Los resultados obtenidos se clasifican en dos grupos. En el primero de ellos se muestran las características sociológicas de los usuarios entrevistados: nacionalidad, sexo, edad y profesión. En el segundo grupo se proporcionan las necesidades de información de los usuarios con respecto al sitio web del archivo.

La figura 5 recoge la nacionalidad de los 90 usuarios entrevistados, en la cual se puede observar que la mayoría $(57 \%)$ son portugueses. En menor medida encontramos brasileños $(9,7 \%)$, estadounidenses $(5,4 \%)$ y españoles $(4,3 \%)$. El resto de usuarios proceden de Alemania, Cabo Verde, Francia, Guatemala e Inglaterra. Tres de los usuarios tienen doble nacionalidad: inglesa-canadiense, luso-brasileña y franco-lusa.

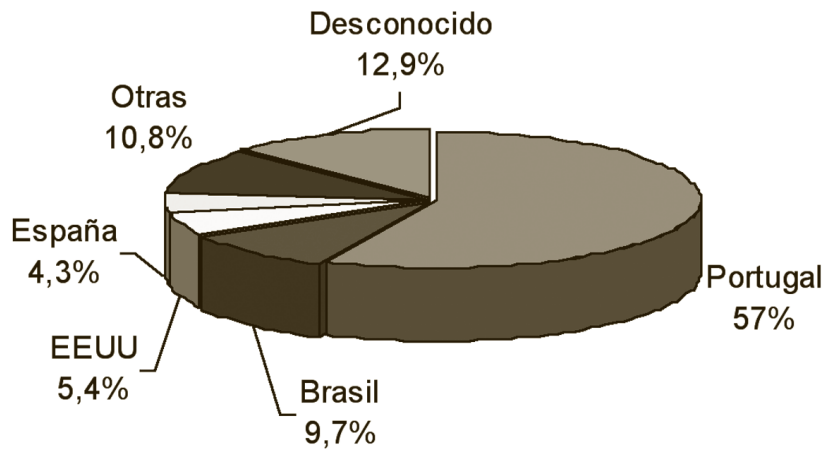

Fig. 5: Nacionalidad de los usuarios entrevistados

La figura 6 (página siguiente) muestra la edad de los usuarios, y en ella se refleja que aproximadamente la mitad $(51,1 \%)$ tienen una edad comprendida entre los 40 y los 60 años, mientras que sólo 7 usuarios $(7,8 \%)$ son mayores de 60 años. Un dato significativo es que 29 usuarios $(32,2 \%)$ tienen menos de 40 años, lo que demuestra que el número de personas jóvenes que utilizan el archivo está incrementándose con el paso del tiempo. A los usuarios se les pidió que se incluyeran en uno de estos tres grupos de edad antes mencionados. 


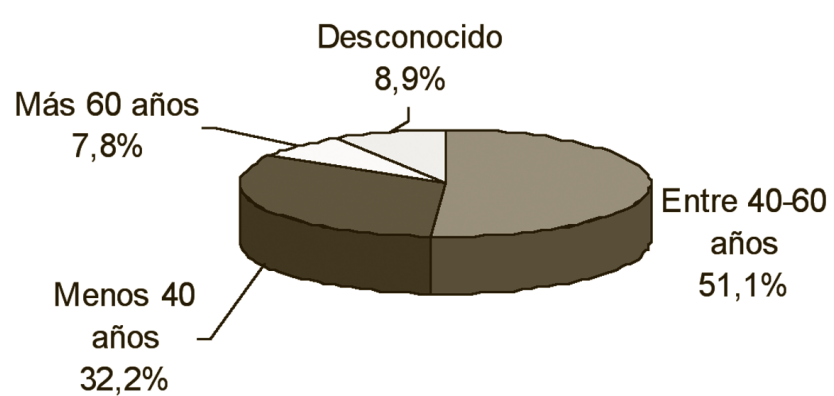

Fig. 6: Edad de los usuarios entrevistados

En la figura 7 se puede apreciar que hay un porcentaje similar de hombres (52\%) y de mujeres (48\%) que utilizan el Arquivo Nacional da Torre do Tombo.

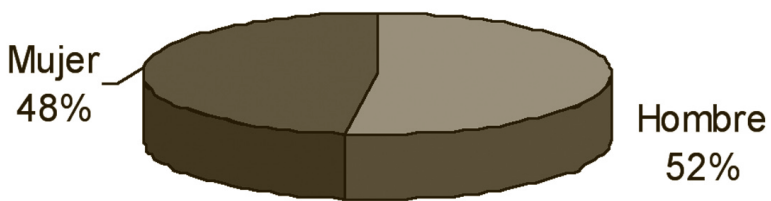

Fig. 7:Usuarios entrevistados, porcentaje comparativo entre hombres y mujeres

La figura 8 (página derecha) muestra la profesión de los usuarios entrevistados, siendo ésta bastante variada. El colectivo más numeroso es el de los profesores (26 usuarios, que representa el 28,9\% del total). De éstos, siete son profesores de secundaria, dieciséis son universitarios y tres no lo especifican. Los investigadores componen el segundo colectivo de usuarios más numeroso del archivo (16,7\%), siendo la investigación en Historia la predominante. Nueve usuarios $(10 \%)$ son estudiantes, por lo que éstos forman otro grupo importante. Tres de ellos estudian la licenciatura de Historia y seis son estudiantes de Doctorado (en Arquitectura, Historia y Lingüística). En menor medida (6 usuarios) encontramos historiadores ${ }^{22}$ y jubilados. Bajo el epígrafe "Otras" se encuentran las profesiones de los otros 14 usuarios (15,6\%): una ama de casa, una antropóloga, dos archiveros, una asistente en la facultad, tres funcionarios públicos, un jornalero, un autónomo, un jurista y tres personas que trabajan en un museo (una conservadora, una técnico superior y otra que no lo especifica).

22 Los usuarios se autodefinieron como historiadores y no como investigadores. Esta distinción también queda de manifiesto entre los usuarios del Arrchivo Nacional de Cuba, y por ello se ha mantenido tal y como estaba. 


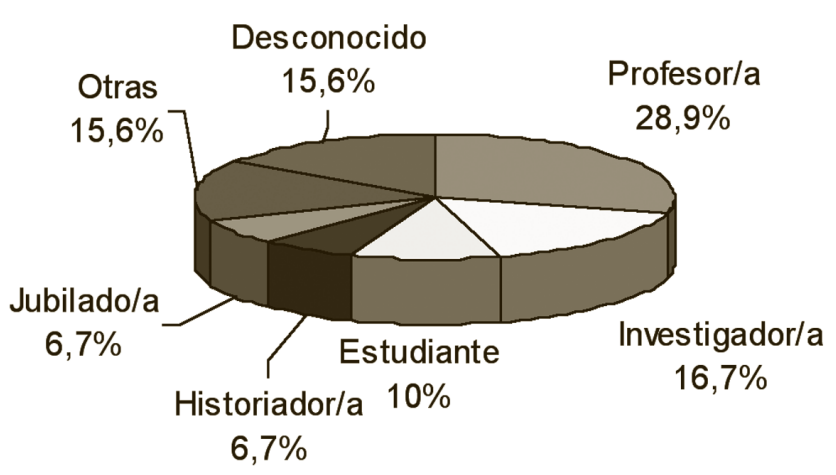

Fig. 8: Profesión de los usuarios entrevistados

Esta diversidad de profesiones nos hace ver el cambio que se está produciendo en los archivos y en la tipología de sus usuarios. Aunque la mayoría de las personas que consultan el archivo son profesores, historiadores e investigadores, hay que tener presente que éstos no son los únicos, hay ciudadanos que no pertenecen a ninguno de estos colectivos que también están interesados en el patrimonio conservado en este tipo de instituciones documentales. Porcentajes ofrecidos sobre los usuarios de otros archivos nacionales, tales como el Archivo Nacional de Cuba (figura 9) reflejan la misma diversidad, y aunque evidentemente los porcentajes no coinciden, sí que hay mucha similitud entre los colectivos que más utilizan esta institución. La muestra es de 990 usuarios que visitaron el Archivo entre los años 1996 y 1999.

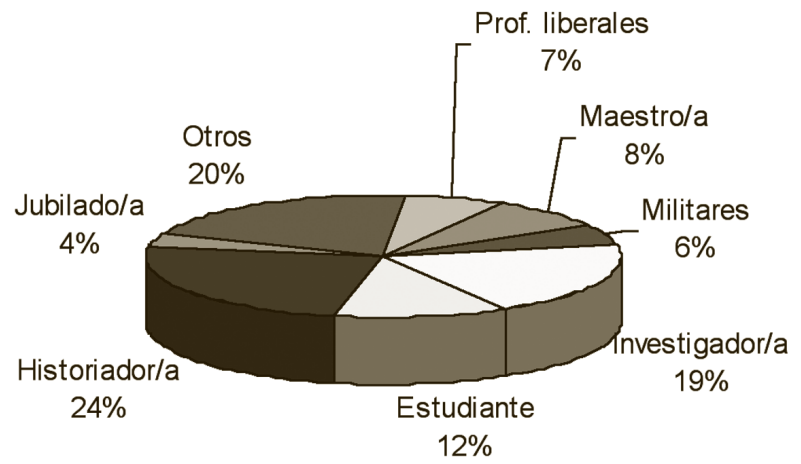

Fig. 9. Profesión de los usuarios del Archivo Nacional de Cuba.

Fuente: Salabarría, Berarda y Gutiérrez, Enma Rita. Características de la relación usuario-servicio en el Archivo Nacional de Cuba. En: Seminário Internacional de Arquivos de Tradição Ibérica [en línea], 3 a 7 de abril de 2000 - Río de Janeiro. [Consulta: 20-11-2008].

Disponible en <http://www.conarq.arquivonacional.gov.br/Media/publicacoes/ibericas/ carac terizacin_de_la_relacin_usuario.pdf $>$ 
Volviendo a Portugal, la figura 10 refleja cuáles son las peticiones más demandas por los usuarios entrevistados con respecto al sitio web del archivo. Se observa que lo más solicitado es tener en el sitio web información sobre los fondos archivísticos, ya sea a través de índices, guías, inventarios o catálogos $(45,6 \%)$, y que comparado con los datos recogidos tres años antes $(39,3 \%)$ supone, como es de esperar, un aumento de usuarios partidarios de recibir servicios a través de la web. La segunda petición más demandada es la posibilidad de consultar el máximo número posible de documentos a través de la web (42,2\%); sin embargo, no coinciden en la temática. Para unos usuarios se deben digitalizar los documentos más consultados, para otros los que ya están microfilmados y para otros los relacionados con su tema de investigación: Inquisición, cronología, cancillerías, actividad comercial, cartografía, Ultramar, Salazar, archivos de la policía, Santo Oficio, genealogía y, sobre todo, los archivos parroquiales.

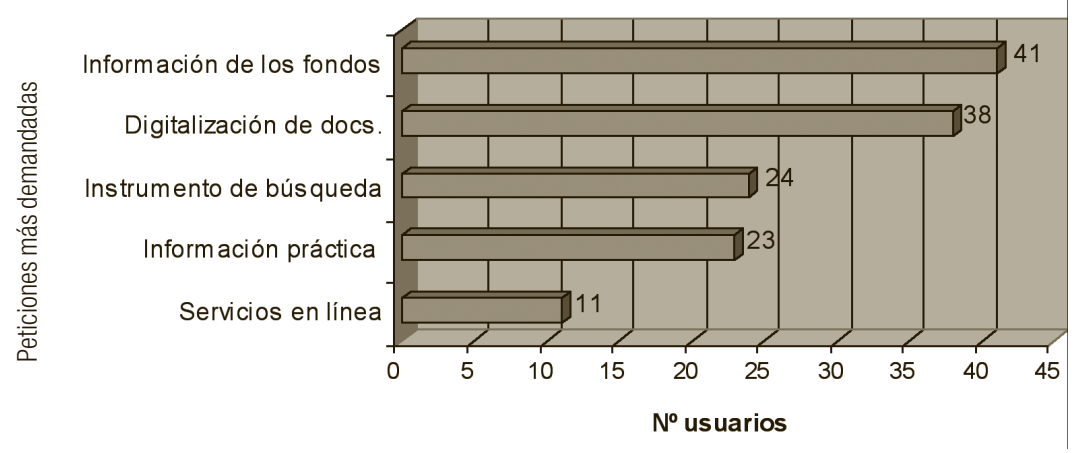

Fig. 10: Peticiones más demandadas por los usuarios entrevistados

Veinticuatro usuarios $(26,7 \%)$ hacen referencia a un instrumento de consulta de fondos (base de datos o catálogo), o a aspectos relacionados con él. Según ellos, las características que debe poseer este instrumento de consulta son que:

- Sea sencillo, claro y fácil de utilizar;

- Esté homogenizado, esto es, que no recoja un mismo autor o documento de maneras diferentes;

- Incluya una descripción de la documentación que recoge;

- Permita hacer consultas sólo en los últimos documentos incorporados desde cierta fecha, de forma que no aparezcan nuevamente los documentos que ya ha consultado;

- Sea detallado, es decir, que incluya el nombre del documento, resumen, fecha y referencia topográfica; 
- Incluya información sobre cómo realizar las búsquedas;

- Haya relaciones entre los documentos;

- Exista integración con otros archivos o instituciones, esto es, que se indique en qué otras instituciones puede encontrar documentos relacionados con una materia determinada;

- Se ofrezca la posibilidad de cruzar datos de varios campos.

Un 25,6\% de los usuarios entrevistados considera necesario incluir en la sede web del archivo información básica o práctica, lo que es, en concreto, la información relacionada con los horarios, transportes (cómo llegar al archivo), normas de acceso y consulta, reglamento interno (qué se puede llevar al archivo, tiempo máximo de consulta, cuál es el procedimiento que se tiene que seguir para solicitar un documento, formas de hacer solicitudes, tiempo que van a demorar en entregarle la documentación, información relativa a la reprografía de los documentos, etcétera) e información similar.

La inclusión de servicios en línea es la última de las peticiones más demandadas, realizadas por 11 usuarios entrevistados (12,2\%), donde se destaca el interés por la existencia de un servicio que permita realizar solicitudes de documentos a través del sitio web. Los otros servicios en línea demandados son:

- Reserva de documentos;

- Alargar el plazo de devolución de la documentación entregada para consulta;

- Solicitud de reprografía de documentos;

- Compra de libros (y otros documentos u objetos) disponibles en la librería/tienda;

- Contacto a tiempo real con los archiveros.

Son servicios que permitirían mejorar en gran medida la comunicación de los usuarios con el archivo. Algunos de los usuarios proponen ideas bastantes interesantes, como la de incluir un listado de los documentos microfilmados y la posibilidad de pedir la reprografía de cualquiera de ellos a través de un enlace; o la de ofrecer una herramienta (como el chat o la video conferencia) que permita estar en contacto a tiempo real con los archiveros.

Consideramos que la puesta en marcha de estos servicios en línea no supondría un descenso de las visitas de los usuarios presenciales sino un aumento de los usuarios virtuales. Barroso y Castillo $^{23}$ lo demuestran en una

23 Anabella Barroso Arahuetes y Cristina Castillo Pérez. "La creación de un servicio digital de archivo como medio de difusión activa: el caso del AHEB-BEHA", en: X Jornadas Españolas de Documentación [CD-Rom]. Santiago de Compostela: FESABID, 2007, p. 85-94. 
obra en la que explican la puesta en marcha de un servicio digital de archivo y en la que constatan que, además de no disminuir el número de usuarios presenciales, éstos cursan sus peticiones a través de la web. Por tanto, estos servicios en línea se convierten en una forma de mejorar los servicios prestados a los usuarios habituales y de captar otros nuevos. El Archivo General de la Nación de México ${ }^{24}$ también ha observado un incremento en el número de accesos a su web. Para el año 2007 programaron 4.5 millones de accesos y al cierre del ejercicio contabilizaron 5.1 millones de accesos (que representan $13.4 \%$ adicional a lo programado).

Sin embargo, el alto número de usuarios que demandan documentación digitalizada, un instrumento de consulta o servicios en línea, contrasta con el hecho de que el 10 por ciento de los usuarios entrevistados utilicen nada o muy poco el sitio web. Los motivos son dos: prefieren ir directamente al archivo (motivo predominante) y porque en el sitio web no suelen encontrar lo que buscan. De esas personas que prefieren ir al Archivo, en lugar de visitar la web, tres son jubilados, dos tienen entre 40 y 60 años, y tres son menores de 40. Del total de personas entrevistadas un 25 por ciento son mujeres, y son todos de ámbitos humanísticos, tanto por su formación como por el tema en el que trabajan en el Archivo (genealogía, derecho, historia y sociología). No se los hemos preguntado, pero existe la posibilidad de que un porcentaje importante de estos usuarios prefieran asistir personalmente a la institución documental debido a malas experiencias previas o por simple desconocimiento de las posibilidades que se ofrecen con las TIC. Otra evidencia que nos hace pensar que no se usa por desconocimiento es que los usuarios más jóvenes son los que están más interesados en que se ofrezca el mayor número de servicios en el sitio web (documentos digitalizados, servicios y catálogos en línea, etcétera), mientras que los mayores buscan en la web lo que solían encontrar como servicios presenciales en los archivos: inventarios, catálogos, etcétera, y apuestan porque se ofrezcan herramientas de descripción lo más completas posible.

Otros productos o servicios que a algunos de los usuarios entrevistados les gustaría que se proporcionara en el sitio web del archivo, por orden de preferencia, son:

- Información sobre el archivo, su historia y sus funciones;

- Transcripciones, así como bibliografía, de obras que conserva el archivo con

24 Archivo General de la Nación de México. Informe anual del Archivo General de la Nación correspondiente al ejercicio 2007 [en línea]. [Consulta: 25-11-2008]. Disponible en <http://www. agn.gob.mx/menuprincipal/quienesomos/informesagn/pdf/informe_anual_agn_2007.pdf>. 
- documentación transcrita;

- Sección con exposiciones y otros eventos culturales;

- Listado de los documentos u objetos disponibles en la librería del archivo;

- Boletín del archivo en un lugar destacado;

- Organigrama y una lista completa de la plantilla del personal, para saber con quién

- contactar;

- Información sobre los nuevos fondos que adquiere el archivo;

- Información clara sobre qué documentos están digitalizados;

- Exposiciones virtuales;

- Breve descripción de los contenidos de las diferentes cajas;

Respecto a las características del sitio web, los usuarios indican que éste debe ser fácil de utilizar, rápido e intuitivo, de manera que la consulta de información sea sencilla. También indican que el sitio web debe estar disponible al menos en otra lengua, especialmente en inglés, y que si se cambia la dirección electrónica (URL), el sistema debería redirigirlos automáticamente. No les gusta que la sede web esté muchas veces (o durante un largo periodo de tiempo) sin funcionar o en reestructuración y que los enlaces no funcionen. Indican además que se debería incluir un apartado 'Novedades' con los últimos contenidos proporcionados en el sitio web (los que estén digitalizados y los que no).

Por otra parte, aunque no se refiere expresamente a la sede web, cabe señalar que dos usuarios resaltan la necesidad de tener red inalámbrica o wi-fi en todas las salas del archivo.

\section{Conclusiones}

No existe unanimidad sobre el concepto y funciones de un Archivo Nacional, ${ }^{25}$ a pesar de que encontramos distintas concepciones existe una función clara admitida de manera unánime: la de conservar la memoria histórica, así como los testimonios de la identidad forjada a través de los siglos. Sin embargo, difícilmente las definiciones de archivo lo asocian con la difusión,

25 Una definición válida es la que se recoge en el Diccionario del archivero-bibliotecario: "archivo histórico que recoge y conserva la documentación relacionada con la historia de la nación”. Otra se recoge en el Diccionario enciclopédico de ciencias de la documentación (edición 2004, p. 101) y lo define como aquella "institución que custodia y gestiona fondos documentales permanentes producidos por las personas físicas y jurídicas, públicas y privadas de un país, región o nación”. 
el uso y el disfrute de todos los ciudadanos de un país a los que debe ser útil. No hay duda de que si la memoria no se conoce, por mucho que se conserve, no se podrá apreciar, incluso es como si no existiera; por ello, facilitar el acceso a las colecciones del archivo a través del sitio web manteniendo, por supuesto, su integridad, tácita o explícitamente late dentro de los deberes de estas magníficas y extensas instituciones públicas que han estado dormidas o en letargo durante mucho tiempo, siendo sólo utilizadas por un grupo minoritario. ${ }^{26}$ Los usuarios ya han manifestado claramente su intención de utilizar las nuevas formas de acceso a la información archivística. Otra cosa muy distinta es que en su fase actual de inmersión en Internet, esto sea o no una prioridad o que, como hemos mantenido en estudios previos para el caso de los archivos nacionales hispanoamericanos,$^{27}$ se esté formando lentamente.

Somos concientes de que la muestra de estudio no es muy amplia. Nos encontramos con el inconveniente de que fue muy escaso el número de usuarios que utilizaron el archivo durante los meses en el que se realizó la investigación, especialmente en agosto. La muestra aquí recogida supone algo menos del 7 por ciento de los usuarios que durante el año 2008 visitaron el ANTT. A pesar de que es reducida, los resultados de la encuesta se asemejan mucho a la realizada en el año 2005 a 210 usuarios, con la salvedad, que por otra parte era de esperar, de que en la que aquí presentamos los porcentajes sobre los partidarios de acceder a información, productos y servicios virtuales es cada vez mayor.

Por todo ello consideramos que sería demasiado atrevido generalizar los resultados obtenidos para cualquier Archivo Nacional, y más aún a cualquier tipo de archivo. Por eso esperamos que este trabajo sirva para animar a otros investigadores a realizar estudios de características similares y así poder contrastarlos. Será una forma más de concienciar a todos los responsables sobre la necesidad, conveniencia y utilidad de prestar cada vez más y mejores servicios virtuales en las instituciones archivísticas, y de acercar éstos a las necesidades reales de sus usuarios.

El sitio web de un archivo puede ser uno de los principales medios de información y difusión del mismo, pero también una herramienta que permita mejorar la comunicación entre usuarios y profesionales de la información, y no sólo porque sea posible contactar con el personal del archivo mediante

26 Celia Chaín Navarro y Amalia Más Bleda. "Bibliotecas y Archivos Nacionales Hispanoamericanos en la Red: hacia la difusión global de la educación y de la memoria histórica", en: Alma América. Libro-Homenaje al profesor Victorino Polo. Murcia: Servicio de Publicaciones, 2008.

27 Amalia Más Bleda, Juan José Sánchez Baena y Celia Chaín Navarro. "Los archivos nacionales de la América hispana y su inmersión en Internet. Presencia, contenidos y disponibilidad informativa", en Revista Nuevo Mundo. Mundos Nuevos, 2007. (Revista electrónica francesa trilingüe). Disponible en $<\mathrm{http}$ ://nuevomundo.revues.org/index9633.html>. 
teléfono, e-mail o formularios electrónicos, sino porque permite ofrecer servicios de diversa tipología en línea, y de este modo acercar el archivo a los ciudadanos. El patrimonio documental que en ellos se custodia debe ser conocido, utilizado y disfrutado por el mayor número posible de usuarios, que muestran una aceptación cada vez mayor hacia el acceso remoto a ellos, ya que actualmente disponemos de las herramientas adecuadas. Es, pues, muy necesario que políticos, gestores, archiveros y/o documentalistas e informáticos nos pongamos a trabajar sobre ello.

Tanto la sede web del archivo como los servicios electrónicos que se ofrezcan en él se deben diseñar desde la perspectiva del usuario. Éstos demandan información relacionada con el archivo (horario, cómo llegar, organigrama, normas de acceso y consulta, etc.) y con los fondos (guías, inventarios, catálogos o cualquier instrumento que les permita conocer en detalle el acervo documental del archivo), pero también están demandando que se facilite la comunicación archivo-usuario: desean herramientas que permitan estar en contacto en tiempo real con los archiveros.

Sobre todo los usuarios solicitan que los archivos permitan que ahorren esfuerzo, tiempo y dinero para satisfacer sus necesidades mediante el empleo de las nuevas tecnologías: quieren documentación digitalizada, solicitan un instrumento de búsqueda sencillo y fácil de utilizar para localizar rápidamente temas de interés, así como desean un servicio archivístico virtual preparado para hacer trámites en línea.

Tengamos en cuenta las necesidades de los usuarios y centremos nuestro trabajo en satisfacerlas de la mejor forma posible.

\section{BiBLIOGRAFÍA}

Archivo General de la Nación de México. Informe anual del Archivo General de la Nación correspondiente al ejercicio 2007 [en línea]. [Consulta: 25-11-2008]. Disponible en <http://www.agn.gob.mx/ menuprincipal/quienesomos/informesagn/pdf/informe_anual_ agn_2007.pdf>.

Barroso Arahuetes, Anabella y Castillo Pérez, Cristina. "La creación de un servicio digital de archivo como medio de difusión activa: el caso del AHEB-BEHA", en: X Jornadas Españolas de Documentación [CD-Rom]. Santiago de Compostela: FESABID, 2007, p. 85-94.

Bonal Zazo, José Luis y Ortego de Lorenzo-Cáceres, María Pilar. "Archivos en línea: formatos de difusión archivística en Internet”, en: VI Jornadas Españolas de Documentación. Valencia: FESABID, 1998, p. 169-182, ISBN: 84-331-4609-X. 
Calva González, Juan José. "La investigación sobre las necesidades de información en comunidades de usuarios”, en Investigación bibliotecológica. 2004, vol. 18, n. 37, p. 23-55.

Conselho Nacional de Arquivos. Diretrizes gerais para a construção de websites de instituições arquivisticas [en línea]. 2000. [Consulta: 26-11-2008]. Disponible en <http://www.portalan.arquivonacional.gov.br/Media/conarqwebsites.pdf $>$.

Cullen, Rowena. Perspectives on User Satisfaction Surveys. Library Trends. 2001, vol. 49, n. 4, p. 662-686.

Chaín Navarro, Celia y Más Bleda, Amalia. "Bibliotecas y Archivos Nacionales Hispanoamericanos en la Red: hacia la difusión global de la educación y de la memoria histórica”, en: Alma América. Libro-Homenaje al profesor Victorino Polo. Murcia: Servicio de Publicaciones, 2008.

Ferrada Cubillos, Mariela. "La satisfacción del usuario remoto de la biblioteca”, en Biblios [en línea]. 2005, n. 21-22. [Consulta: 01-112008]. Disponible en <http://eprints.rclis.org/archive/ 00004709/>.

García Delgado, Purificación. "El servicio de referencia digital", en El profesional de la información. 2004, vol. 12, n. 4, p. 320-330.

González Teruel, Aurora y Abad García, Francisca. "Information needs and uses: an analysis of the literature published in Spain, 1990-2004", en Library and information science reseach [en línea]. 2007, vol. 29, n. 1, p. 30-46. [Consulta: 07-08-2008]. Disponible en <http://eprints.rclis.org/archive/00012136/01/Information_ needs_and_uses.pdf $>$.

Hassan Montero, Yusef. "Factores del diseño web orientado a la satisfacción y no-frustración de uso", en Revista Española de Documentación Científica. 2006, vol. 29, n. 2, p. 239-257.

Martín Fernández, Francisco Jesús. La Experiencia del Usuario. No Solo Usabilidad [en línea]. 2005, n. 4. [Consulta: 0110-2008]. Disponible en <http://www.nosolousabilidad.com/articulos/experiencia_del_usuario.htm $>$.

, Martín Fernández, Francisco Jesús e Iazza, Ghzala. "Diseño Web Centrado en el Usuario: Usabilidad y Arquitectura de la Información", en Anuario Hipertext.net [en línea]. 2004, n. 2. [Consulta: 01-10-2008]. Disponible en <http://www.hipertext. net/web/pag206.htm>.

Hernández Salazar, Patricia. "La relación entre los estudios y la formación de usuarios de la información”, en Revista General de Información y Documentación. 2007, vol. 17, n. 2, p. 103-121.

International Federation of Library Associations (IFLA). Recomendaciones para el Servicio de Referencia Digital [en línea]. 2006. [Consulta: 02-09-2008]. Disponible en <http://www.ifla.org/VII/s36/ pubs/drg03-s.htm>

Lara Navarra, Pablo y Ruiz Rodríguez, Antonio. "La difusión de información en los archivos: páginas web”, en Tria. Revista de la Asociación de Archiveros de Andalucía. 1999, n. 6, p. 151-163. 
Más Bleda, Amalia; Sánchez Baena, Juan José y Chaín Navarro, Celia. "Los archivos nacionales de la América hispana y su inmersión en Internet. Presencia, contenidos y disponibilidad informativa”, en Revista Nuevo Mundo. Mundos Nuevos, 2007. (Revista electrónica francesa trilingüe). Disponible en <http://nuevomundo.revues. org/index9633.html>.

Manso Rodríguez, Ramón Alberto. Servicio de Referencia Virtual: concepto, organización y evaluación [en línea]. Santa Clara (Cuba): Editorial Feijoo, 2006. [Consulta: 05-11-2008]. Disponible en $<$ http://eprints.rclis.org/archive/00015079/>.

"Servicio de referencia virtual: teoría y práctica en torno a las políticas para su gestión y desarrollo", en Revista Española de Documentación Científica. 2008, vol. 31, n. 1, p. 39-51.

Marcos Mora, Mari Carmen. Interacción en interfaces de recuperación de información: conceptos, metáforas y visualización. Gijón: Trea, 2004, 354 p.

Martín Moreno, Carmen. Metodología de investigación en estudios de usuarios. Revista General de Información y Documentación. 2007, vol. 17, n. 2, p. 129-149.

Martín Suquía, Ramón. "Sistemas de recuperación de la información en los archivos: un análisis de situación y perspectivas”, en Lligall. 2001, n. 17, p. 47-72.

Muñoz, María Rosa de. "Usuarios internos y externos en los archivos administrativos de Panamá”, en: Seminário Internacional de Arquivos de Tradição Ibérica [en línea], 3 a 7 de abril de 2000 - Río de Janeiro. [Consulta: 20-11-2008]. Disponible en <http://www.conarq.arquivonacional.gov.br/Media/publicacoes/ibericas/usuarios_internos_y_externos_en_los_archivos_administrativos_de_ panama.pdf $>$.

Navarro Bonilla, Diego. "El servicio de referencia archivístico: retos y oportunidades”, en Revista Española de Documentación Cientifica. 2001, vol. 24, n. 2, p. 178-197.

Olvera-Lobo, María Dolores y Benítez-de-Vendrell, Belarmina. "Aproximación a las actitudes y percepciones de los usuarios ante las tecnologías de la información”, en El profesional de la información. 2008, vol. 17, n.2, p. 199-204.

Rey Martín, Carina. "La satisfacción del usuario: un concepto en alza”, en Anales de Documentación. 2000, vol. 3, p. 139-153.

Rodríguez Briz, Fernanda. Los servicios de referencia virtual: surgimiento, desarrollo y perspectivas a futuro. Buenos Aires: Alfagrama, 2005, $156 \mathrm{p}$.

Romero Martín, Felipe. "User experience: modelos mentales y expectativas”, en: Knapp Bjerén, Alberto (coord.). La experiencia de usuario. Madrid, Anaya: 2002, p. 327-341. 
Rubio Hernández, Alfonso. "Estudios de usuarios en archivos municipales: una aproximación teórico-práctica”, en Scire. Representación y Organización del Conocimiento. 2004, vol. 10, n. 1, p. $217-$ 236.

Salabarría, Berarda y gutiérrez, Enma Rita. "Características de la relación usuario-servicio en el Archivo Nacional de Cuba”, en: Seminário Internacional de Arquivos de Tradição Ibérica [en línea], 3 a 7 de abril de 2000 - Río de Janeiro. [Consulta: 20-11-2008]. Disponible en <http://www.conarq.arquivonacional.gov.br/Media/publicacoes/ibericas/caracterizacin_de_la_relacin_usuario.pdf $>$.

Santaella Ruiz, Rita Dolores. "Los archivos históricos provinciales: estudio de usuarios aplicado en Jaén”, en Revista General de Información y Documentación. 2006, vol. 16, n. 2, p. 155-167.

, "Usuarios de información pública de la ciudad de Jaén (1976-1996)", en Revista General de Información y Documentación. 2007, vol. 17, n. 2, p. 77-94.

y Ruiz Rodríguez, Antonio Ángel. Estudio de usuarios de información aplicado al archivo: la ciudad de Jaén. Jaén: Universidad de Jaén, 2007, 169 p.

Sanz Casado, Elías. "La realización de estudios de usuarios: una necesidad urgente", en Revista General de Información y Documentación. 1993, vol. 3, n. 1, p. 154-166.

, Manual de estudios de usuarios. Madrid: Fundación Germán Sánchez Ruipérez, 1994, 288 p. , y Martín Moreno, Carmen. "Técnicas bibliométricas aplicadas a los estudios de usuarios", en Revista General de Información y Documentación. 1997, vol. 7, p. 41-68.

Sridhar, M. S. "Understanding the user - why, what and how?", en $\mathrm{Li}$ brary Science with a slant to Documentation and Information Studies [en línea]. 1995, vol. 32, n. 4, p. 151-164. [Consulta: 16-10-2008]. Disponible en <http://eprints.rclis.org/archive/00009340/>.

Torrés Rosell, Antoni. Márquetin y archivos. Propuestas para una aplicación del márquetin en los archivos. Gijón: Trea, 2006, 191 p.

Torres Vargas, Georgina Araceli y Sánchez Avillaneda, María del Rosario. "El servicio de referencia en la biblioteca digital", en Investigación bibliotecológica. 2005, vol. 19, n. 39, p. 120-133.

Valdehita Mayoral, María Teresa. "Los usuarios en el archivo general de la UNED”, en Revista General de Información y Documentación. 2007, vol. 17, n. 2, p. 31-44. 